\title{
Radical solutions
}

\author{
The US RADx program has spawned a phalanx of diagnostic products to market in just 12 months. Its long-term \\ impact on point-of-care, at-home and population testing may be even more profound.
}

I ver the past year, the US National Institutes of Health (NIH) Rapid Acceleration of Diagnostics (RADx) program has invested hundreds of millions of dollars into the development of new diagnostic products. RADx funding and expertise has supported $>150$ companies and launched 16 diagnostic products, with another 15 in the pipeline; overall, the program has put 125 million COVID-19 tests onto the market since April 2020. It has also spurred development of a slew of new technologies-including handheld PCR devices, loop-mediated amplification tests, paper-based diagnostics, rapid lateral flow assay (LFA) antigen tests, smartphone readers, next-generation sequencing (NGS) and machine-learning-assisted diagnostics-in a matter of months. This combination of RADx technologies, together with structural changes to healthcare during the pandemic, has the potential to radically change diagnostics, opening up the point-of-care (POC), at-home and community testing settings.

RADx was established by the NIH at the end of April 2020 as part of $\$ 1.5$ billion appropriated for SARS-CoV-2 testing by US Congress in the Paycheck Protection Program and Health Care Enhancement Act. The US National Institute of Biomedical Imaging and Bioengineering established programs to build testing capacity for school and university reopening (RADx Tech and RADx ATP), galvanize innovative diagnostic and surveillance development (RADx-rad) and jump-start efforts to reach vulnerable and underserved populations (RADx-UP). By matching developers with experts from a pool of $\sim 600$ academicians, entrepreneurs and regulators, RADx aims to not only galvanize simultaneous development of assay and devices, but also parallelize performance assessment, regulatory interactions, manufacturing capacity and supply-chain logistics to compress into a single year what is typically a five-year product development cycle.

To date, RADx has awarded a total of \$520 million in 27 contracts (whittled down from a starting set of 716 applications) complementing another $\sim \$ 157$ million in funding from the Biomedical Advanced Research and Development Authority. And, with the December passage of the Consolidated Appropriations Act, 2021 and signing of the American Rescue Plan Act of 2021 last month, another $\$ 71.55$ billion is being made available for testing, contact tracing, surveillance and containment in the coming year.

These are eye-popping numbers, especially when one considers that the entire global market for clinical diagnostics in 2020 was just $\$ 56$ billion. The question is whether, postpandemic, the diagnostic sector will revert to its status of 'ugly stepchild' of healthcarewith centralized laboratories, entrenched giants like Quest and LabCorp, low margins and sales, and risible investment from the private sector-or whether RADx and other investments can effect lasting change.

In certain clinical settings, RADx technology promises to change medical practice. For example, as COVID-19 becomes endemic, handheld devices developed by Mesa Biotech or Mammoth Biosciences could speed patient triage in emergency rooms, enabling rapid distinction among viruses causing respiratory infections, such as SARS-CoV-2, influenza $\mathrm{A}$ or $\mathrm{B}$, and respiratory syncytial virus. Similarly, greater uptake of molecular tests in clinical microbiology can supersede culturing approaches carried over from the nineteenth century, returning lab results in minutes or hours rather than days.

But it is the $\$ 29.5$ billion POC market (using trained personnel in physician offices and pop-up labs) and the massively underpenetrated at-home direct-to-consumer (DTC) market that seem likely to see the most change.

The RADx program is supporting numerous POC applications, including 14 PCR tests and 7 LFA antigen tests. The use of artificial intelligence for pattern recognition of test readouts and to support non-experienced technicians in areas like ultrasound will also broaden market opportunities. Similarly, Medicare reimbursement for COVID-19 testing will drive test uptake, even if private payer coverage remains variable.

Post-pandemic, increasing use of telehealth and remote care is likely to further drive diagnostics into community or home settings. In December, RADx awardee Ellume's multiplex quantum dot fluorescence test and smartphone app received Emergency Use Authorization (EUA) for home use. The app allows test data readout to be automated and returned to the physician or other provider, illustrating how home testing can be connected to the healthcare infrastructure as digital medicine increasingly intersects with diagnostics. Last month, another EUA was given to a Cue Health home test kit with a reusable cartridge reader and app, opening the door to repeat home testing.

This also has implications for low- to middle-income markets, where sustained underinvestment means that basic diagnostics have been limited to just $1 \%$ of primary care facilities and $14 \%$ of hospitals. As smartphones are already widely available in many countries, digitally enabled diagnostics could expand testing to millions more people in remote settings lacking clinical infrastructure-although the digital divide remains a concern.

A final area where RADx has targeted funding is the use of NGS platforms as an early warning system for potential outbreaks. Surveillance can be used for spot sampling of surfaces, air, urban wastewater and long-haul flight waste. The use of sample pooling is likely to prove extremely useful in opening schools and screening employees. It will also galvanize testing for SARS-CoV-2 variants circulating in the population and enable test, trace and isolate efforts during community transmission.

These trends lead to an unexpected collision of previously disparate diagnostic realms. NGS already has a foothold in clinical settings, steering therapeutic interventions via multiplexed assays for cancer, infectious agents, antimicrobial resistance genes and microbiome profiling. If the slew of funding for surveillance bears fruit outside COVID-19, the divisions between public health surveillance and individual-patient-oriented clinical diagnostics may start to blur.

Overall, RADx has both radically shifted the funding available for innovative diagnostics and greatly foreshortened product development times. But it will all be for naught if the current outmoded one-test, one-person paradigm isn't exchanged for a robust infrastructure and rational reimbursement system that actually empowers community testing and diagnostic-led medicine. For too long, we have talked the talk of precision medicine. Now is the time to walk the walk.

Published online: 6 April 2021 https://doi.org/10.1038/s41587-021-00908-5 